\title{
CLASSIFICATION OF OSTEOPOROSIS BASED ON TEXTURE FEATURES
}

\author{
P.Prabakaran, G.Manikannan, T.Vijayalakshmi \\ Assistant Professor, Department of Electronics and Communication Engineering, \\ CK College of Engineering and Technology, Cuddalore.
}

\begin{abstract}
There was substantial challenge in assessment of osteoporotic disease from the radiograph image. Texture characteristics are visually very analogous when witnessed from the naked eye for the bone micro- architecture of the osteoporotic and healthy cases which will be a challenging grouping problem. An approach that is based on a combination of multi resolution Gabor filters and 1D local binary pattern (1DLBP) features is proposed to extract the discriminative patterns in all the orientations and scales simultaneously. Gabor filter are used due to their advantages in yielding a scale and orientation sensitive analysis whereas LBPs are useful for quantifying microstructural changes in the images. The proposed method shows good classification results with an overall accuracy of about $\mathbf{7 2 . 7 1 \%}$.
\end{abstract}

Index Terms - Gabor filters, Osteoporosis, Pattern recognition, Texture analysis.

\section{INTRODUCTION}

Osteoporosis is an age-related growing problem in the world [1]. Although an awareness about the concerns for osteoporosis is increasing day by day, the diagnosis and prevention methods are still inadequate. Osteoporosis is a skeletal disease that affects the bone strength and makes them susceptible to the fractures. This disease is described by damage of bone mass and the weakening bone mi- croarchitecture [2]. Thus, bone mass is not the only aspect of importance, the skeletal factors and micro-architecture features are also of great significance [3]. Bone Mineral Density (BMD) is a degree of mineral content present in the bone and is an exceptional pointer for osteoporosis. BMD is typically defined by the Dual Energy X-ray Absorptiometry (DXA) [4]. However, using only BMD is unsatisfactory for guessing the fracture risk precisely [5]. It has been verified that evaluating osteoporosis using bone mass is unclear [6]. Combining the bone microarchitecture features with BMD coefficients provides an excellent way for preventing fracture risk. Although there are many other technologies like Trabecular Bone Score but none of them prove to be very useful in clinical practice [7]. According to many studies [8], [9] the texture analysis of bone radiographs is a promising way to assess and identify this disease effectively. Many techniques use three dimensional (3D) non-invasive measures to assess the bone structure. Despite the accuracy of these methods, these are not very practical due to their complexity, price, and access to the devices. 2D texture analysis presents a simpler way to evaluate the bone structure using radiographs [9]. The 2D texture analysis is an indirect assessment of 3D micro-architecture [10]. This assists in extracting the information about the bone structure and helps in separating the healthy from the osteoporotic cases. Many studies have been conducted based on two Dimensional (2D) bone radiographs using several texture analysis meth- ods [11]. Fractal geometry is based on fractal dimension to characterize the textures displaying irregular and self- similarity behaviors. Fractal analysis has been proved as a suitable tool to quantify the bone structure changes due to the fractal nature of bone [12]. This analysis can be done using the fractional Brownian motion ( $\mathrm{fBm})$ and its increments, fractional Gaussian noise (fGn) [13]. Lacunarity analysis has also been proposed for the osteoporosis detection [14]. Gabor transform and wavelets have also been used to study the bone changes [15]. Some investigations have been made using the morphological factors for bone structure analysis [16]. Hybrid methods combining statistical and structural methods like Local Binary Pattern (LBP) operator have been proposed that have the ability to identify the changes within the trabecular bone [17]. A directional multi-scale analysis has been performed to study the bone texture characterization and calcaneus radiographs have been analysed using circular parametric models [18]. In [19], a study has been conducted which used special feature selection techniques that take into account the individual prediction ability of the feature and the inter-feature redundancy to find the most distinct feature set.

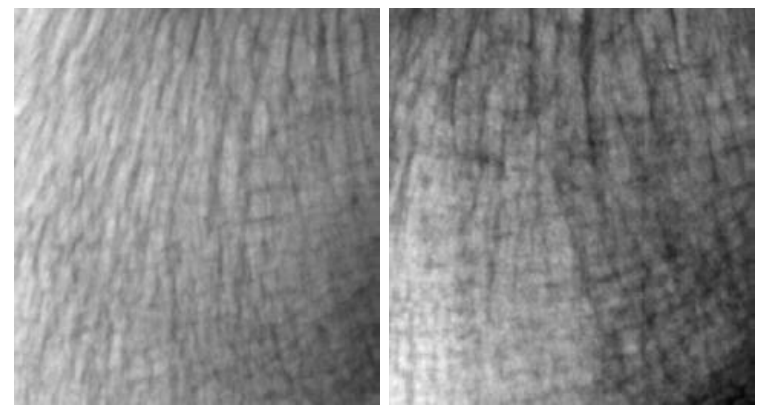

Fig. 1: Normal (left) and Abnormal (right) X-ray images.

Diagnosis of osteoporosis using bone radiographs presents a great challenge because the texture exhibited by the healthy and osteoporotic cases 


\section{International Journal of Engineering Applied Sciences and Technology, 2019 Vol. 4, Issue 8, ISSN No. 2455-2143, Pages 208-212 \\ Published Online December 2019 in IJEAST (http://www.ijeast.com)}

are visually very similar to each other (Fig. 1).In this paper, we have proposed a method for the automated diagnosis of osteoporosis in bone radiographs. Our premise is that the structural changes in trabecular bone can be defined by textural features, as established in the literature [19]. We present an approach combining Gabor filters and one Dimensional (1D) LBP that provides significant discriminating features. Briefly, this approach is based multi-resolution spatial analysis combining intensity distribution with spatial information. Instead of using directly using spatial intensity distribution, multiple scales and orientations of the Gabor filters followed by 1D LBP operator are used. The combination of Gabor and 1D LBP improved the representation power of spatial histogram and provided features that enhance different shapes of the structure. Our goal is to take benefit from both the Gabor and 1D LBP to extract an effective descriptor that can improve the performance for the early stage diagnosis of osteoporosis. The paper is organized as follows: we discuss the dataset used (Section II) followed by the description of the proposed method (Section III). Later, we describe our experimental results (Section IV) followed by conclusive remarks (Section V).

\section{MATERIALS}

All the experiments are conducted on Texture Characterization of Bone (TCB) challenge data publicly available at http://www.univ-orleans.fr/i3mto/data. This data consists of healthy and osteoporotic radiograph images. Any experiments involving human beings were approved by the Review Board of the institution that provides the data. The data set includes the annotated images of 58 healthy and 58 osteoporotic subjects. The region of interest (ROI) was marked by a physician by pointing out the anatomical marks that can be simply recognized on the calcaneus image. This guarantees that the ROI be picked up in the same area and in the same alignment from each bone radiography. ROI was of $2.7 \times 2.7 \mathrm{~cm} 2$ positioned in a part of the calcaneus that comprises of only the bone. The pixel size was $105 \mathrm{~m}$. Figure 1 presents four sample images. The top images are from osteoporotic patients and the bottom two are from the healthy subjects. These calcaneus images present an interesting dataset, since all of them show similar visual characteristics.

\section{METHODS}

The visual illustration of a region of interest in the radiograph of a heel is shown in Fig. 2. The heel is subjected to two forces, gravity (tension) and walking force (compression) which create anisotropic properties in the bone structure. Moreover, there is a different in granularity of the bones for normal (dense) and osteoporotic bones (scale changes). Therefore, the multiresolution analysis using Gabor filters (orientation and scale variations) and microstructural analysis (local binary patterns) becomes very interesting.

A. Gabor filtering

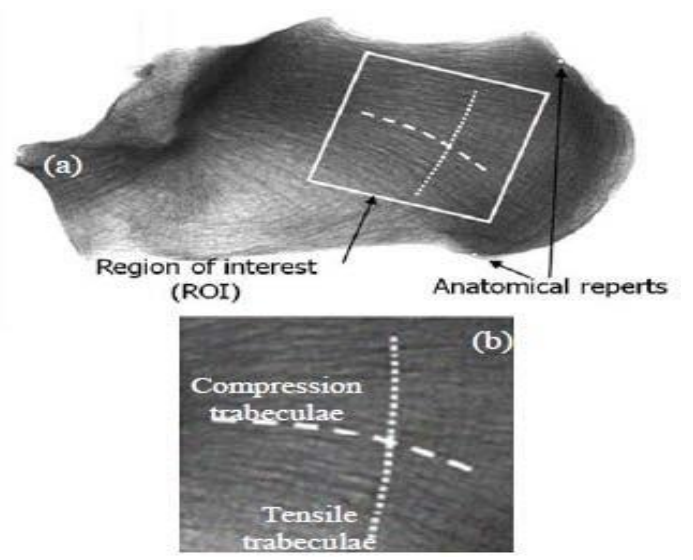

Fig. 2: Radiograph with its region of interest (adapted from [20].

The osteoporosis images are subjected to feature extraction using a combination of Gabor filters and Local Binary Patterns (LBP). Over the recent years, Gabor filters have been used widely to address various issues related to texture classification. The usage of Gabor filters is motivated by the fact that they are very similar with the primary mammalian visual cortex [21]. Another significant consideration is that the Gabor filters achieve optimal localization in both frequency and spatial domain [21]. The Gabor filtering of an image is represented as

$$
\begin{aligned}
& G \theta, \sigma(x, y)=I(x, y) * \psi \theta, \sigma(x, y) \\
= & \int_{k-\infty}^{\infty} \int_{l=-\infty}^{\infty} I(x, y) \psi \theta, \sigma(x-k, y-l)--(1)
\end{aligned}
$$

where $\mathrm{I}(\bullet)$ denotes the input images, $\psi \theta, \sigma(\bullet)$ represents the impulsive response of a Gabor filter having an orientation $\theta$ and scale $\sigma$ whereas $*$ represents the operator of convolution. A Gabor filter can be mathematically defined as follows:

$$
\begin{aligned}
& \psi(x, y)= \frac{f^{2}}{\pi \gamma \eta} e^{\left(\frac{f^{2}}{\gamma^{2}} x^{\prime 2}+\frac{f^{2}}{\eta^{2}} y^{\prime 2}\right)} e^{j 2 \pi f x^{\prime}}-- \\
& X^{\prime}=x \cos \theta+y \sin \theta \\
& Y^{\prime}=-x \sin \theta+y \cos \theta
\end{aligned}
$$

where $\mathrm{f}$ is the centre frequency of the filter, $\theta$ is the orientation of the major axis of a Gaussian function, $\gamma$ is the standard deviation along the major axis and $\eta$ is standard deviation along the minor axis. $\lambda=\eta \gamma$ represents the aspect ratio of the Gaussian function. A 2D Gabor filter function in the frequency domain is written as

$$
\begin{aligned}
& \Psi(u, v)=e^{\frac{-\pi^{2}}{f^{2}\left(\gamma^{2}\left(u^{\prime}-f\right)^{2}+\eta^{2} v^{\prime 2}\right)}--(4)} \\
& u^{\prime}=u \cos \theta+v \sin \theta \\
& v^{\prime}=-u \sin \theta+v \cos \theta
\end{aligned}
$$




\section{International Journal of Engineering Applied Sciences and Technology, 2019 Vol. 4, Issue 8, ISSN No. 2455-2143, Pages 208-212 \\ Published Online December 2019 in IJEAST (http://www.ijeast.com)}

which represents a bandpass filter. A bank of Gabor filters is obtained by changing the parameters of a Gabor waveletand obtaining the relevant filter banks. If $\mathrm{S}$ is the total number of scales and $\mathrm{K}$ is the total number of orientations at which the Gabor filters are calculated, we obtain a feature vector of size $\mathrm{S} \times \mathrm{K}$ for every pixel in a Gabor filtered image.

\section{B. 1D Projections of Filtered Responses}

The filtered images are subjected to the calculations of their projections along the horizontal and vertical axis. As a result of this projection, we can potentially find out how the variations are taking place either horizontally or vertically in the images. This is a very reasonable strategy to calculate the variations in the images given that the subject changes will be reflected in the response of the relevant filter bank. Given filter output $\mathrm{G} \theta, \sigma(\mathrm{x}, \mathrm{y})$, its horizontal and vertical projections are calculated as

$$
G_{\theta, \sigma}^{H}=\sum_{i=0}^{\text {Rows }} G_{\theta, \sigma}(x, y), G_{\theta, \sigma}^{V}=\sum_{i=0}^{\text {Cols }} G_{\theta, \sigma}(x, y)
$$

\section{1D Local Binary Patterns (LBP)}

The projection vectors are now subjected to $1 \mathrm{D}$ LBPs. The LBPs were first proposed by Ojala et al. [22] and then later have been widely used for the texture feature extraction. The state-of-the-art representation of LBP used eight neighboring pixels for the neighborhood information and used the center pixel as the threshold value to encode the other pixel information. The $2 \mathrm{D}$ LBP is not very useful for bone texture characterization because bone structure mainly exhibits anisotropic changes. The 2D LBP captures only the frequency of the local structures and have no consideration for their global orientation and thus is less sensitive to anisotropic changes. An illustration for the basic concept of 1DLBP that we have adopted is given in Fig. 3. After the calculation of 1DLBPs for every filter bank both along the horizontal and vertical directions, we calculate the histograms of LBP which can be used as features for the images.

\section{Dimensionality reduction}

For feature reduction, we have performed principal component analysis (PCA). The PCA decomposes the data such that the features are organized in the order of decreasing variance values of the decomposed components of the signal. Effectively, PCA can be used to fit the data on an n-dimensional space where the value of _ $\mathrm{n}_{-}$is chosen such that a specific percentage of variance of the data is incorporated in the transformed feature space.

\section{E. Classification}

We have chosen different statistical classifiers in our work, their choice is chosen by their main archetypes: Nearest Neighbor: This classifier labels an unknown object with the label of the majority class of its'k' neighbors in the feature space [24]. The distance between an object and its nearest neighbors is measured in the Euclidean sense.

Support Vector Machines: The SVM, originally proposed by Vapnik et al. [25] mainly consists of constructing an optimum hyperplane that maximizes the margin of separation between two different classes. This approach typically constructs the classification models which have excellent generalization ability thus making it a powerful tool in various applications. For our implementation, we have used SVM with linear kernel for classification.

\section{EXPERIMENTS}

In this section, all the experiments are reported on the TCB challenge data. At first, the Gabor filter bank consisting of filter of four scales and four orientations are constructed. Later, 1DLBP histograms are obtained. For the calculation of LBPs, 8 neighbors are considered at a spacing of 1 pixel. Feature extraction is followed by feature reduction using PCA, keeping about 95\% variance of the data. Classification is done using two well-known classifiers: the 1 Nearest Neighbours (1NN) and Support Vector Machine (SVM). A ten-fold cross validation is performed to evaluate the performance of our method. In this work we first compared our method using LBP and 1DLBP. The mask is an important parameter determining the classification performance. Thus, the results for LBP with 8 neighbours and 1DLBP with 8 neighbours are used.

We compared our method with the technique proposed in [17]. We replicated the method and compiled the results for all 116 images of the data set for $1 \mathrm{NN}$ and SVM. A part from this technique that exists in the literature with reference to osteoporotic image classification, we compared the performance obtained using two other state-of-the-art texture feature extraction methods i.e., homogeneous texture [26] and local binary patterns [22]. Our experiments show that the proporsed method outperforms the other methods that have been considered in this paper, irrespective of the classifier used. We suspect that this is because of the multiresolution and multidirectional nature of the proposed descriptor as compared to ther other counterparts that have been used. Al- though the HT is also a multiresolution descriptor, it exhibits a shortcoming of the aggregation of the filter responses thus ignoring the details in the images. 


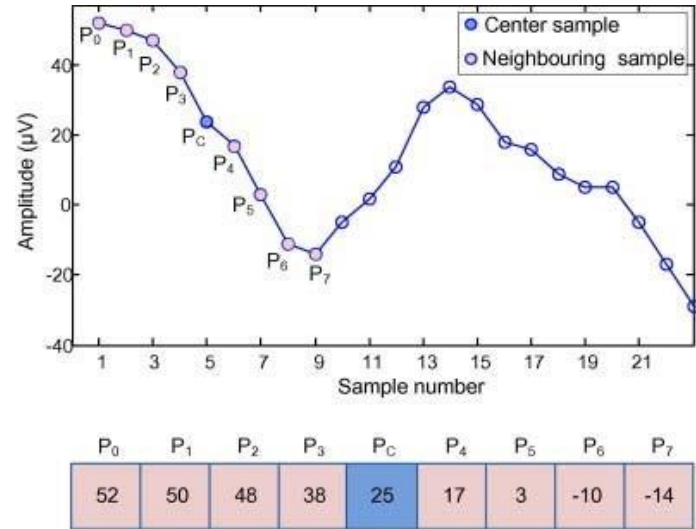

(a)

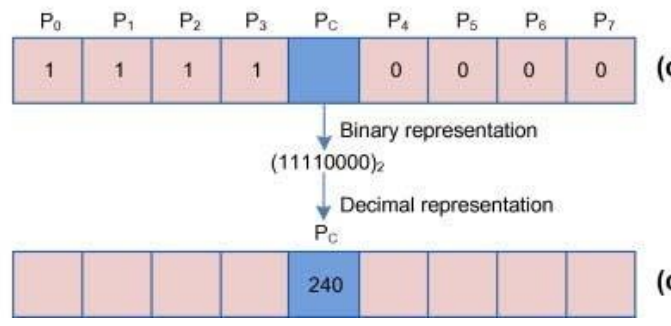

Fig. 3: The principle of obtaining 1D Local Binary Patterns (adapted from [23]).

Although reasonable results are obtained, it should be noted that there is a scope for improvement in the results. We have not adopted the more conventional ways of using the convolutional neural networks (CNN) in this paper because we face several challenges, the most important one being that the dataset pertaining to osteoporosis is limited (only 118 images, half from normal bones and half from osteoporotic bones).

\begin{tabular}{|c|c|c|}
\hline Method & SVM & KNN \\
\hline HT & 62.57 & 64.18 \\
\hline LBP & 59.64 & 60.25 \\
\hline Wavelet-1DLBP & 67.94 & 68.62 \\
\hline Proposed & $\mathbf{7 0 . 5 9}$ & $\mathbf{7 2 . 7 1}$ \\
\hline
\end{tabular}

TABLE I: Detection Results for Osteoporosis.

\section{CONCLUSIONS}

In this paper, we have proposed a novel technique for the extraction of texture features from X-Ray radiographs. The method is based on two state-of-theart texture feature extraction methods: Gabor filters and local binary patterns which exhibit their own distinct advantages. The texture features are obtained using Gabor filtering first, followed by the calculation of the projected Gabor responses (along hori- zontal and vertical directions), obtaining two vectors for each filtered output. This output is subjected to the calculation of 1DLBPs followed by feature reduction and classification of the images. It should be noted that the strengths of both LBP (microstructure analysis) and Gabor filters (multiresolution analysis) are utilized in the proposed features. The idea is to capture multi- resolution global and local characteristics patterns to achieve a better characterization of the images. Our results show that the proposed feature set shows good results, although there is still scope for improvement.

There are several issues with this study, which are mainly triggered by the lack of adequate data. Due to this limitation, it is not very feasible to use more sophisticated classification methods such as CNNs on the images (unless proper data augmentation is done). In the future, we would like to address this shortcoming by systematically augmenting the dataset or collecting more dataset from the practitioners to ensure broader conclusions for the subject scenario.

\section{REFERENCES}

[1] A. H. Warriner and K. G. Saag, (2013) "Osteoporosis diagnosis and medical treatment," Orthopedic Clinics, vol. 44, no. 2, pp. 125-135.

[2] J. Hays, J. K. Ockene, R. L. Brunner, J. M. Kotchen, J. E. Manson, R. E. Patterson, A. K. Aragaki, S. A. Shumaker, R. G. Brzyski, A. Z. LaCroix et al. (2003), "Effects of estrogen plus progestin on healthrelated quality of life," New England Journal of Medicine, vol. 348, no. 19, pp. 1839-1854.

[3] L. Dalle Carbonare and S. Giannini, (2004) "Bonemicroarchitecture as an important determinant of bone strength," Journal of endocrinological investigation, vol. 27, no. 1, pp. 99-105.

[4] G. M. Blake and I. Fogelman, (2010) "An update on dual-energy x-ray absorptiometry," in Seminars in nuclear medicine, vol. 40, no. 1. Elsevier, pp. 62-73.

[5] S. R. Cummings and D. Black, (1995) "Bone mass measurements and risk of fracturein caucasian women: A review of findings from prospective studies," The American journal of medicine, vol. 98, no. 2, pp. 24S$28 \mathrm{~S}$.

[6] S. Schuit, M. Van der Klift, A. Weel, C. De Laet, H. Burger, E. Seeman, A. Hofman, A. Uitterlinden, J. Van Leeuwen, and H. Pols, (2004) "Fracture incidence and association with bone mineral density in elderly men and women: the rotterdam study," Bone, vol. 34, no. 1, pp. 195-202.

[7] E. Lespessailles, C. Gadois, I. Kousignian, J. Neveu, P. Fardellone, S. Kolta, C. Roux, J. Do-Huu, and C. Benhamou, (2008) "Clinical interest of bone texture analysis in osteoporosis: a case control multicenter study," Osteoporosis international, vol. 19, no. 7, pp. 1019-1028.

[8] L. Pothuaud, E. Lespessailles, R. Harba, R. Jennane, V. Royant, E. Eynard, and C.-L. Benhamou, (1998) "Fractal analysis of trabecular bone texture on radiographs: discriminant value in postmenopausal osteoporosis," Osteoporosis International, vol. 8, no. 6, pp. 618-626.

[9] A. Taleb-Ahmed, P. Dubois, and E. Duquenoy, (2003) "Analysis methods of ct-scan images for the 


\section{International Journal of Engineering Applied Sciences and Technology, 2019 Vol. 4, Issue 8, ISSN No. 2455-2143, Pages 208-212 \\ Published Online December 2019 in IJEAST (http://www.ijeast.com)}

characterization of the bone texture: First results," Pattern recognition letters, vol. 24, no. 12, pp. 19711982.

[10] T.-H. Hou and M.-D. Pern, (1999) "A computer vision-based shape- classification system usingimage projection and a neural network," The International Journal of Advanced Manufacturing Technology, vol. 15 , no. 11 , pp. 843-850.

[11]F. Mallard, B. Bouvard, P. Mercier, P. Bizot, P. Cronier, and D. Chap- pard, (2013) "Trabecular microarchitecture in established osteoporosis: relationship between vertebrae, distal radius and calcaneus by X-ray imaging texture analysis," Orthopaedics \& Traumatology: Surgery \& Research, vol. 99, no. 1, pp. 52-59.

[12] R. Jennane, R. Harba, G. Lemineur, S. Bretteil, A. Estrade, and C. L. Benhamou, (2007), "Estimation of the $3 \mathrm{~d}$ self-similarity parameter of trabecular bone from its 2d projection," Medical Image Analysis, vol. 11, no. 1 , pp. 91-98,

[13] T. Lundahl, W. J. Ohley, S. M. Kay, and R. Siffert, (1986), "Fractional brownian motion: A maximum likelihood estimator and its application to image texture," IEEE Transactions on medical imaging, vol. 5, no. 3, pp. 152-161.

[14] A. Zaia, R. Eleonori, P. Maponi, R. Rossi, and R. Murri, (2005) "Medical imaging and osteoporosis: Fractal's lacunarity analysis of trabecular bone in $\mathrm{mr}$ images," in Computer-Based Medical Systems, 2005. Proceedings. 18th IEEE Symposium on. IEEE, pp. 38.

[15] J. Pramudito, S. Soegijoko, T. Mengko, F. Muchtadi, and R. Wachjudi,( 2007 ), “Trabecular pattern analysis of proximal femur radiographs for osteo- porosis detection," Journal of Biomedical \& Pharmaceutical Engineer-ing, vol. 1, no. 1, pp. 45-51.

[16] S. Sevestre-Ghalila, A. Benazza-Benyahia, A. Ricordeau, N. Mellouli, C. Chappard, and C. L. Benhamou, "Texture image analysis for osteoporosis detection with morphological tools," in International Conference on Medical Image Computing and Computer-Assisted Intervention. Springer, 2004, pp. 87-94.

[17] L. Houam, A. Hafiane, A. Boukrouche, E. Lespessailles, and R. Jen- nane, (2014), "One dimensional local binary pattern for bone texture characterization," Pattern Analysis and Applications, vol. 17, no. 1, pp. 179-193,.

[18] H. Oulhaj, M. Rziza, A. Amine, H. Toumi, E. Lespessailles, R. Jen- nane, and M. El Hassouni, (2017), "Trabecular bone characterization using circular parametric models," Biomedical Signal Processing and Con trol, vol. 33, pp. 411-421.

[19] K. Zheng and S. Makrogiannis, "Bone texture characterization for osteoporosis diagnosis using digital radiography, (2016), " in Engineering in Medicine and Biology Society (EMBC), 2016 IEEE 38th Annual International Conference of the. IEEE, pp. 1034-1037. [20] L. Houam, A. Hafiane, R. Jennane, A. Boukrouche, and E. Lespessailles, (2011), "Trabecular bone texture classification using $1 \mathrm{~d} \mathrm{lbp}$ and wavelet coefficients in high-pass bands," in International Conference on Signal, Image, Vision and their Applications SIVA, vol. 11, pp. 21-24.

[21] D. A. Pollen and S. F. Ronner, (1983), "Visual cortical neurons as localized spatial frequency filters," IEEE Transactions on Systems, Man, and Cybernetics, no. 5, pp. 907-916.

[22] T. Ojala, M. Pietikainen, and T. Maenpaa, (2002), "Multiresolution gray-scale and rotation invariant texture classification with local binary patterns," IEEE Transactions on pattern analysis and machine intelligence, vol. 24, no. 7, pp. 971-987.

[23] Y. Kaya, M. Uyar, R. Tekin, and S. Yıldırım, (2014), "1d-local binary pattern based feature extraction for classification of epileptic eeg signals," Applied Mathematics and Computation, vol. 243, pp. 209-219.

[24] K. Fukunaga, (2013), "Introduction to statistical pattern recognition". Elsevier.

[25] V. Vapnik, (2013), "The nature of statistical learning theory" Springer science \& business media.

[26] B. S. Manjunath, J.-R. Ohm, V. V. Vasudevan, and A. Yamada,(2001), "Color and texture descriptors," IEEE Transactions on circuits and systems for video technology, vol. 11, no. 6, pp. 703-715.

\section{ACKNOWLEDGEMENT}

1. Mr.P.Prabakaran, is presently working as an Assistant Professor in the Department of Electronics and Communication Engineering at CK college of Engineering and Technology, Cuddalore. He have completed his UG degree at Krishnasamy College of Engineering and Technology, Cuddalore under the discipline of ECE and completed his PG degree in AC college of Engineering and Technology, Karaikudi, under the discipline of Optical communication.

2. Mr.G.Manikannan, is presently working as an Assistant Professor in the Department of Electronics and Communication Engineering at CK college of Engineering and Technology, Cuddalore. He have completed his UG degree at Aravindar College of Engineering and Technology, Vanur under the discipline of ECE and completed his PG degree in Manakula vinayagar Institute of Technology under the discipline of VLSI.

3. Mrs.T.Vijayalakshmi, is presently working as an Assistant Professor in the Department of Electronics and Communication Engineering at CK college of Engineering and Technology, Cuddalore. She have completed his UG degree at Mailam Engineering College, Mailam under the discipline of ECE and completed his PG degree in Arunai College of Engineering under the discipline of VLSI Design. 